\title{
Burst and Tonic Response Modes in Thalamic Neurons During Sleep and Wakefulness
}

\author{
THEODORE G. WEYAND, MICHAEL BOUDREAUX, AND WILLIAM GUIDO \\ Department of Cell Biology and Anatomy, Louisiana State University Medical Center, New Orleans, Louisiana 70112
}

Received 5 June 2000; accepted in final form 8 December 2000

Weyand, Theodore G., Michael Boudreaux, and William Guido. Burst and tonic response modes in thalamic neurons during sleep and wakefulness. J Neurophysiol 85: 1107-1118, 2001. Thalamic neurons can exhibit two distinct firing modes: tonic and burst. In the lateral geniculate nucleus (LGN), the tonic mode appears as a relatively faithful relay of visual information from retina to cortex. The function of the burst mode is less understood. Its prevalence during slow-wave sleep (SWS) and linkage to synchronous cortical electroencephalogram (EEG) suggest that it has an important role during this form of sleep. Although not nearly as common, bursting can also occur during wakefulness. The goal of this study was to identify conditions that affect burst probability, and to compare burst incidence during sleeping and waking. LGN neurons are extraordinarily heterogenous in the degree to which they burst, during both sleeping and waking. Some LGN neurons never burst under any conditions during wakefulness, and several never burst during slow-wave sleep. During wakefulness, $<1 \%$ of action potentials were associated with bursting, whereas during sleep this fraction jumps to $18 \%$. Although bursting was most common during slow-wave sleep, more than $50 \%$ of the bursting originated from $14 \%$ of the LGN cells. Bursting during sleep was largely restricted to episodes lasting $1-5 \mathrm{~s}$, with $\sim 47 \%$ of these episodes being rhythmic and in the delta frequency range $(0.5-4 \mathrm{~Hz})$. In wakefulness, although visual stimulation accounted for the greatest number of bursts, it was still a small fraction of the total response $(4 \%, 742$ bursts/17,744 cycles in 93 cells). We identified two variables that appeared to influence burst probability: size of the visual stimuli used to elicit responses and behavioral state. Increased stimulus size increased burst probability. We attribute this to the increased influence large stimuli have on a cell's inhibitory mechanisms. As with sleep, a large fraction of bursting originated from a small number of cells. During visual stimulation, $50 \%$ of bursting was generated by $9 \%$ of neurons. Increased vigilance was negatively correlated with burst probability. Visual stimuli presented during active fixation (i.e., when the animal must fixate on an overt fixation point) were less likely to produce bursting, than when the same visual stimuli were presented but no fixation point present ("passive" fixation). Such observations suggest that even brief departures from attentive states can hyperpolarize neurons sufficiently to de-inactivate the burst mechanism. Our results provide a new view of the temporal structure of bursting during slow-wave sleep; one that supports episodic rhythmic activity in the intact animal. In addition, because bursting could be tied to specific conditions within wakefulness, we suggest that bursting has a specific function within that state.

\section{IN T R O D U C T I O N}

Owing to similarities in receptive field structure, the lateral geniculate nucleus (LGN) appears to largely function as a relay

Address for reprint requests: T. G. Weyand, Dept. of Cell Biology and Anatomy, Louisiana State University Medical Center, 1901 Perdido St., New Orleans, LA 70112 (E-mail: tweyan@1suhsc.edu). of visual information between the retina and visual cortex. However, it is not a simple relay. Anatomical studies indicate that $<20 \%$ of afferent synapses are of retinal origin (see Sherman and Guillery 1996, for recent review). These other inputs likely provide a gain mechanism for controlling the fidelity by which retinal inputs are transferred to cortex (Livingstone and Hubel 1981; Swadlow and Weyand 1985; see reviews by McCormick and Bal 1997; Sherman 1996; Singer 1977) or synchronize activity to boost the salience of spatially contiguous edges (Sillito et al. 1994). Bolstered by observations from intracellular records, several groups (e.g., McCormick and Feeser 1990) have additionally promoted the idea that the LGN operates as a switch. In the tonic or "on" mode, retinal inputs are available for transfer to cortex, with the exact gain being a function of the current potency and sign of nonretinal afferents. In the "burst" or "off" mode, the membrane potential is predominantly hyperpolarized, but occasional (or even periodic) depolarization triggers a powerful low-threshold calcium conductance $\left(I_{\mathrm{T}}\right)$ that results in bursts of action potentials. This burst mode is correlated with slow-wave sleep (SWS). Whereas the function of the tonic mode is transparent, the function of the burst mode is obscure. Steriade et al. (1993) recently speculated that because the burst mode is associated with initiating and maintaining slow-wave sleep, its ability to drive the cortex into oscillations may function to correct some ionic imbalances caused by wakefulness. Although not nearly as frequent, bursting can also occur within wakefulness (Guido and Weyand 1995; McCarley et al. 1983; Ramcharan et al. 2000). Such observations are important, as they raise the possibility that bursting is used as a distinct signal in sensory processing (cf., Guido and Weyand 1995; Guido et al. 1995; Sherman 1996). The conditions under which bursting occurs during wakefulness are not well-delineated. Identifying these conditions was a major goal of the current study. A second goal was to better delineate the incidence of bursting during slow-wave sleep. Several recent and influential reviews have portrayed thalamic neurons as "disconnected" from their sensory inputs during slow-wave sleep, whereupon such neurons become oscillatory as a result of an interplay of their intrinsic conductances (McCormick and Bal 1997; Steriade et al. 1993). Such portrayals are a caricature of the one quantitative study of the temporal structure of LGN activity during sleep and waking (McCarley et al. 1983). A reassess-

\footnotetext{
The costs of publication of this article were defrayed in part by the payment of page charges. The article must therefore be hereby marked "advertisement" in accordance with 18 U.S.C. Section 1734 solely to indicate this fact.
} 
ment of activity during slow-wave sleep might help to clarify this important issue.

\section{MET H O D S}

\section{Initial surgery}

All procedures were approved by the Institutional Care and Use Committees at Louisiana State University Medical Center, and the general methods have been described previously (Malpeli et al. 1992; Weyand and Gafka 1998). Briefly, cats underwent at least two sterile surgical procedures. In the first surgery, we cemented an aluminum crown to the skull (to fix the head during subsequent behavioral and recording sessions) and attached an insulated wire loop to the sclera of one eye (to allow us to determine gaze using the magnetic search coil technique) (Robinson 1962). The anesthetized cat was placed in a stereotaxic frame, and the fascia and muscle retracted to expose the skull. An aluminum crown was custom-fitted to the contours of the skull and affixed to the skull using stainless steel rod and bone cement. A Teflon-insulated coil was then attached to the sclera of one eye similar to the methods described by Judge et al. (1980). The leads from this coil were fed under the skin and crown, and terminated by soldering them to subminiature connectors. A fiberglass cover was attached to the crown to protect the connectors and microelectrode drive when the animal was not in the testing apparatus. The cat was removed from the stereotaxic frame and returned to its home cage. Following training (described in Training), the cat was subjected to a second sterile surgery in which we implanted stimulating electrodes in visual cortex and a swiveling base for holding a microelectrode drive (Malpeli et al. 1992). For this surgery, the anesthetized cat was again placed in a stereotaxic frame, and holes were drilled through the cement and bone to expose visual cortex and the cortex overlying the LGN. Six to eight stimulating electrodes were placed individually into the lateral gyrus $(\sim 1 \mathrm{~mm}$ apart and at depths of $3-5 \mathrm{~mm})$ through an intact dura. The electrodes were cemented into place and the hole sealed with dental acrylic. A microelectrode base with a 9-mm-long cannula was inserted through the hole over the LGN. A protective stylus filled the cannula until we were ready to begin recording sessions. The hole over the LGN was then sealed with dental cement, and the cat removed from the stereotaxic frame and returned to its home cage. To obtain electroencephalographic (EEG) records, we either used a pair of unused stimulating electrodes, or, in one case, we inserted a staggered pair (2-mm vertical separation) of platinuminsulated wires into the contralateral cortex with the upper tip flush with the cortical surface.

\section{Training}

Following at least $1 \mathrm{wk}$ to recover from initial surgery, food deprivation was begun and $24 \mathrm{~h}$ later behavioral testing initiated. The cat was placed in a loose-fitting canvas bag and the head fixed to a Plexiglas frame. The cat faced a dimly lit $\left(0.5 \mathrm{~cd} / \mathrm{m}^{2}\right)$ screen on which we could project images such as bars, square-wave gratings (contrast 0.4 ) and a $0.2^{\circ}$ spot produced by a low-power laser that was dimmed with a 3.0 N.D. filter. The cat was trained to look at this spot, and if it jumped to a new location, make a saccade to reacquire fixation on the spot. The bars and gratings were used to probe the excitability of the cell under study and were never behaviorally relevant. The laser spot was always behaviorally relevant.

\section{Testing/recording}

Following at least $1 \mathrm{wk}$ to recover from surgery to implant stimulating electrodes, testing was initiated by placing the cat in the apparatus and replacing the protective stylus with a tungsten-in-glass microelectrode $(\sim 1.0 \mathrm{M} \Omega$ at $1 \mathrm{kHz})$. Signals from the microelectrode were amplified $(\times 10,000)$, filtered $(0.3-8 \mathrm{KHz}, 24 \mathrm{~dB} /$ octave $)$, and fed to an oscilloscope and audio monitor. EEG signals were also amplified $(\times 10,000)$, filtered $(1-60 \mathrm{~Hz}, 24 \mathrm{~dB} /$ octave), and passed to an oscilloscope and an A/D (A-D) converter that digitized the signals at $250 \mathrm{~Hz}$. Horizontal and vertical eye position signals were amplified, filtered, and passed to an A-D converter that digitized these signals at $250 \mathrm{~Hz}$. All data acquisition, control of behavioral testing, and stimulus display were under computer control. Single neurons were isolated on-line using a voltage discriminator, whose output (pulses) was fed to the computer. These pulses were then time-stamped (0.1-ms resolution) and put into a data file that also included a record of eye position, EEG, and stimulus status. For more than 30 recording sessions, the eye position, EEG signals, and unit activity were also passed to a VCR that digitized data at $22.5 \mathrm{kHz}$. These records were used for off-line analysis and for producing figures of analog traces.

For most sessions, we used electrical stimulation of visual cortex to help determine the position of the electrode in the brain. Electrical stimuli consisted of 0.1-ms monophasic pulses of varying intensity. Electrical activation was attempted for nearly all isolated neurons. Antidromic activation was inferred by invariant latency and verified by the test of impulse collision (Bishop et al. 1962). Some neurons (especially those dorsal to the LGN) were activated synaptically rather than antidromically.

\section{Definition of bursts}

Because we recorded extracellularly and filtered out activity $<300$ $\mathrm{Hz}$, we could not directly observe changes in membrane potential associated with activation of the low-threshold calcium conductance $\left(I_{\mathrm{T}}\right)$. Lu et al. (1992) showed that when two or more action potentials separated by $<4 \mathrm{~ms}(250 \mathrm{~Hz})$ are preceded by at least $100 \mathrm{~ms}$ of quiescence, the probability of an underlying $I_{\mathrm{T}}$ is better than 0.99 . These criteria correspond to what has been used in previous studies of bursting in anesthetized, paralyzed (Guido et al. 1995; Lu et al. 1993), and awake, behaving cat (Guido and Weyand 1995). Although we feel these criteria to be sufficient, our results from activity patterns during sleep indicate that they are overly conservative (cf., Lu et al. 1993; Ramacharan et al. 2000). Therefore in presenting our results, we have sometimes adopted more liberal criteria of two spikes in $6.66 \mathrm{~ms}(150$ $\mathrm{Hz}$ ) preceded by $50 \mathrm{~ms}$ of quiescence. Adapting one criteria or the other obviously alters the quantitative aspects of burst incidence, but does not alter the major conclusions of the study. In each figure and analysis, we indicate the criteria used: conservative (4 $\mathrm{ms}$ or less interspike interval preceded by $100 \mathrm{~ms}$ or more of quiescence) or liberal $(6.66 \mathrm{~ms}$ or less interspike interval preceded by $50 \mathrm{~ms}$ or more of quiescence). Two-dimensional interspike interval plots (joint-interval histograms, JIHs) (McCarley et al. 1983) shown in Figs. 2 and 6 are particularly useful for viewing differences in burst incidence using liberal or conservative criteria. In Fig. 2, we have inscribed two "boxes" in the bottom right corner showing the differences between using conservative (smaller box) or liberal (larger box) criteria. For this representative example, changing criteria had essentially no effect in wakefulness, but increased the number of bursts during slow-wave sleep by $\sim 25 \%$.

\section{Definition of sleep}

Slow-wave sleep was defined by the presence of dominant cortical EEG spectra below $8 \mathrm{~Hz}$. Although delta waves $(0.5-4 \mathrm{~Hz})$ were the most impressive EEG event during slow-wave sleep (e.g., Fig. 1), these waves were interspersed with episodes of faster activity that we also included as slow-wave sleep when accompanied by closed eyes and slow or absent eye movements. Rapid eye movement (REM) sleep was not observed, probably because we never deliberately sleep-deprived our animals, or perhaps because the testing situation was not particularly conducive to sleeping. In addition, sleep spindles (large-amplitude $10-$ to $14-\mathrm{Hz}$ cortical oscillations) were not commonly observed probably because our EEG electrodes were above 

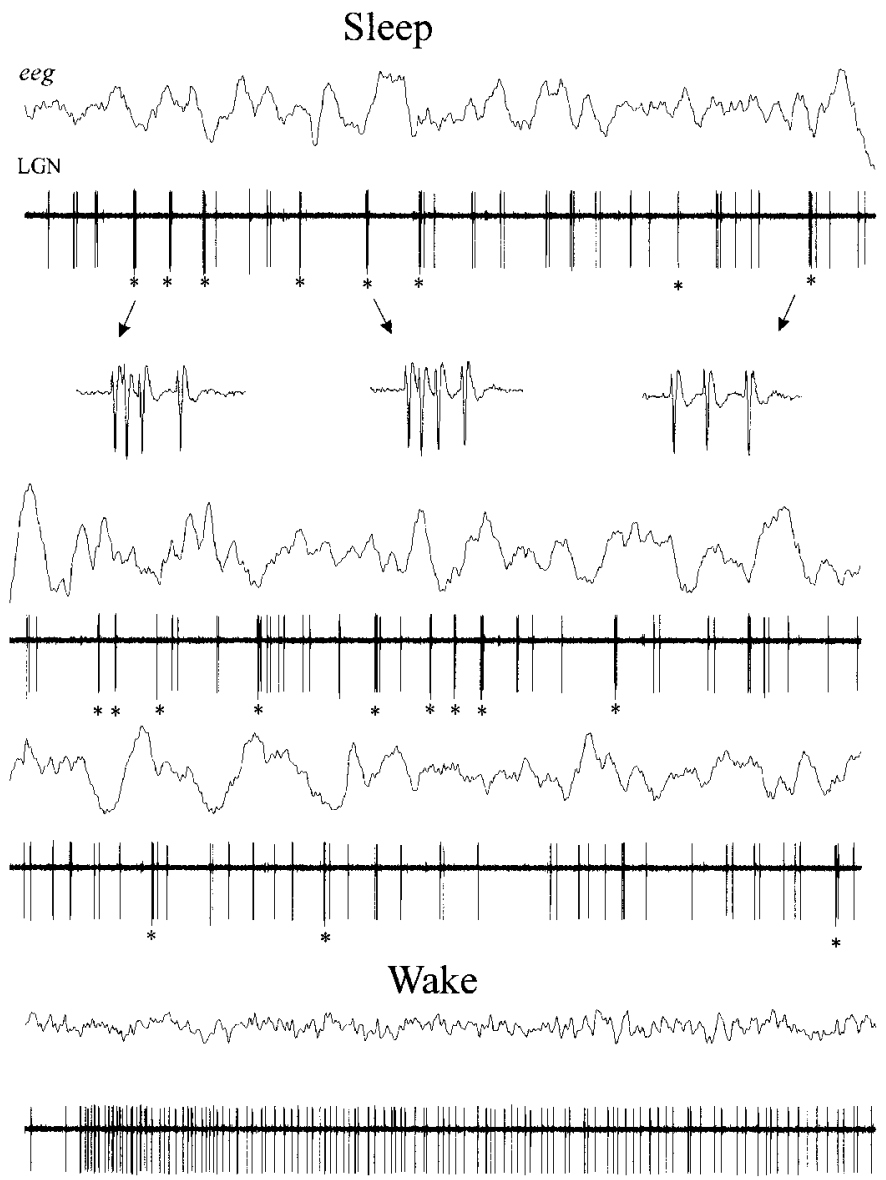

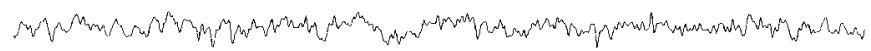
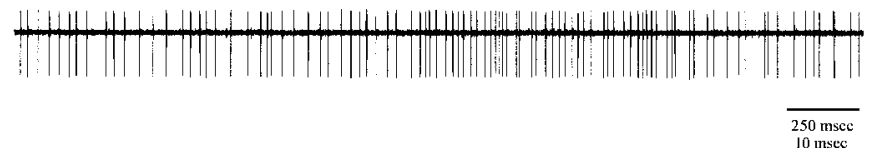

FIG. 1. Simultaneous recordings of occipital cortical electroencephalogram (EEG) and activity of a lateral geniculate nucleus (LGN) neuron during sleep (top 7 traces) and wakefulness (bottom 4 traces). Sleep is dominated by episodic high-frequency bursts (asterisks, liberal criteria), whereas wakefulness is dominated by very regular (tonic) activity. On the $3 r d$ line, 3 of the bursts have been expanded in time to increase the spatial detail of individual action potentials.

occipital cortex, where splindling is less pronounced (cf., Steriade and Llinas 1988).

\section{Analysis of rhythmicity}

Bursting during sleep, while common, was episodic. To facilitate evaluating underlying rhythmicity of bursting within these episodes, we created and analyzed "burst bouts." A burst bout was defined as four or more bursts occurring within $2 \mathrm{~s}$ and interburst intervals $<0.1 \mathrm{~s}$. The onset of the bout was the first spike in the first burst, and the end was defined as the last spike in the last burst. Rhythmicity of these bouts was evaluated by first determining the standard deviation of interburst intervals for each bout. Standard deviation is inversely related to rhythmicity; perfect rhythmicity would yield a standard deviation of 0 . Confidence that a given burst bout was rhythmic was assigned by comparing the rank of the observed standard deviation relative to 999 simulations. The simulations were created by using the same number of observed bursts, but with interburst intervals randomly drawn. The constraint on these random numbers was that they must lie within a range that could have occurred in the observed burst bout. The rank of the value of the observed standard deviation is a direct measure of the reliability in a statistical sense that the observed bout was rhythmic.

\section{R E S U L T S}

We recorded from $148 \mathrm{LGN}$ neurons in three cats. Among these, 109 were identified in layer A, 21 in A1, and 9 in C. In addition, we also encountered nine fibers above the LGN, four of which could be antidromically activated from visual cortex. We assumed that the cell bodies of these fibers were in the LGN, although we cannot be sure of the layer. Because of eye movements in the awake animal, we made no attempt to classify neurons as $\mathrm{X}, \mathrm{Y}$, or $\mathrm{W}$. Twenty-two neurons were identified using antidromic identification. Based on latency, we had a clear recording bias for Y cells (mean latency, $0.91 \mathrm{~ms}$; $\sigma=0.61 \mathrm{~ms}$; range, $0.4-3.0 \mathrm{~ms} ; 15 / 22$ latencies $<1.2 \mathrm{~ms}$ ) (So and Shapley 1979).

\section{Bursts during sleep and waking}

Figure 1 presents analog traces of an LGN neuron and concomitant cortical EEG during sleep (top) and wakefulness (bottom), illustrating the burst and tonic modes commonly associated with these two states. As the name implies, the burst mode is characterized by periods of quiescence interspersed with high-frequency "bursts" of action potentials. Other studies have demonstrated that these bursts are sodium spikes (action potentials) riding a slower depolarization attributed to the activation of the low-threshold calcium current $I_{\mathrm{T}}$. The top records show expanded views of three bursts (conservative criteria). Bursting during sleep tends to be episodic or rhythmic, and we describe some quantitative aspects below. During "tonic" episodes as illustrated in the bottom traces, activity can be remarkably regular, often varying with ambient illumination level. For the traces shown, $95 \%$ of the activity was at $50 \mathrm{~Hz}$ or less. Figure 2 shows the temporal distribution of a different LGN neuron over a prolonged period (10 min) during which the cat drifts in and out of sleep. Figure $2 A$ shows the distribution in raster format with the first spike in a burst indicated by a large dot, whereas Fig. $2 B$ shows the spike distribution as JIHs (McCarley et al. 1983) during each epoch of sleep (left) and wakefulness (right). The JIHs sharply contrast the temporal structure of activity during sleep and wakefulness. During wakefulness, activity is remarkably regular (tonic) and a central distribution is obvious. In contrast, during sleep the regularity is disrupted (burst mode) and two tails form. The tail in the bottom right of each JIH is the cardinal spike of a burst, while the well-defined tail to bottom left indicates the shortest interspike interval (highest frequency) achieved within a burst. Figure $2 C$ shows the sleep/wake cycles collapsed into two JIHs and includes insets to illustrate the differences in burst incidence using the two sets of criteria used in this study. Dots within the smaller inset represent the cardinal spike of bursts (and hence, the number of bursts) using the conservative criteria, whereas dots within the larger inset represent the cardinal spikes of bursts using the liberal criteria. Although these figures illustrate the general temporal structure of many LGN neurons during sleep and wakefulness, the data presented be- 
A

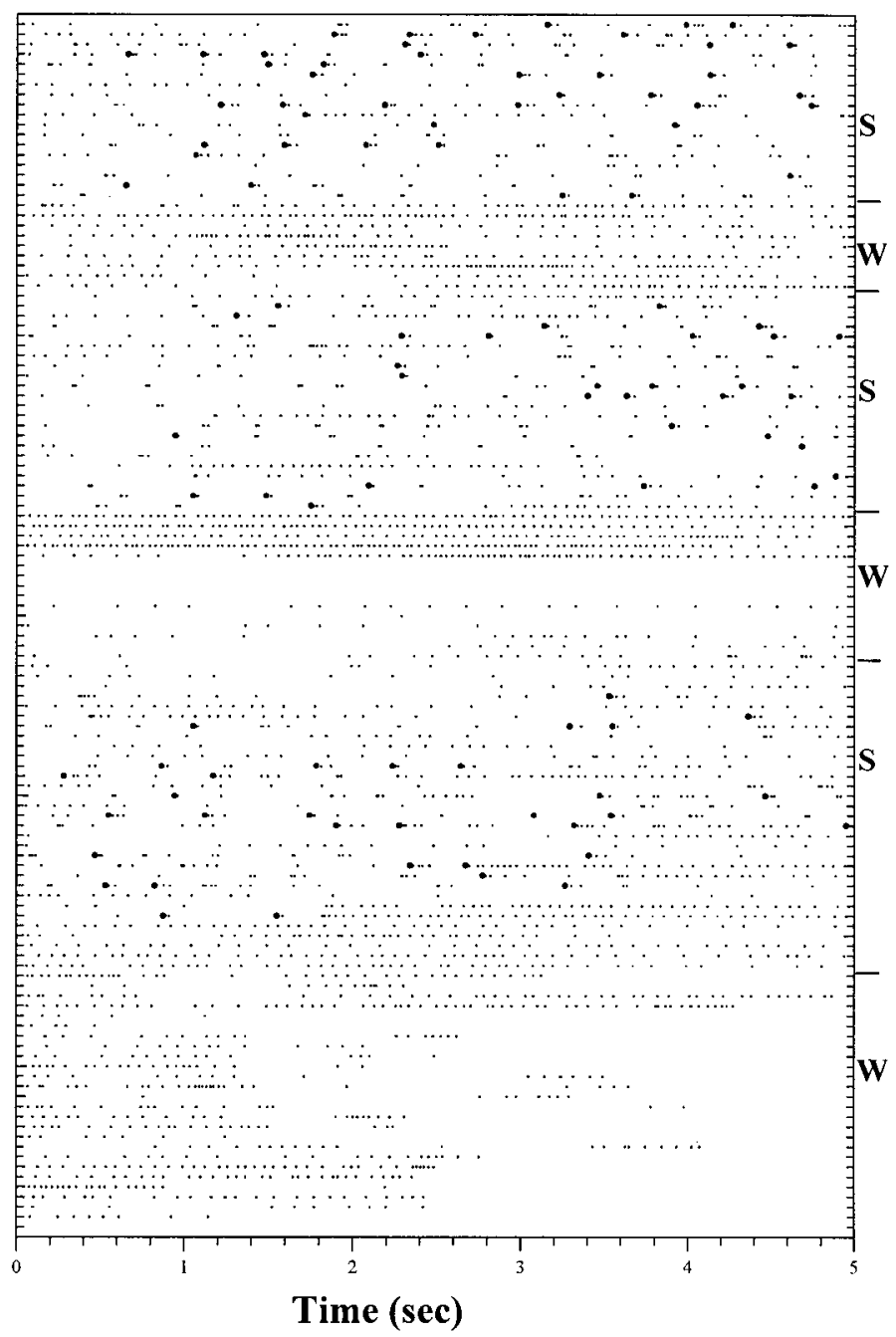

Time (sec)

Sleep

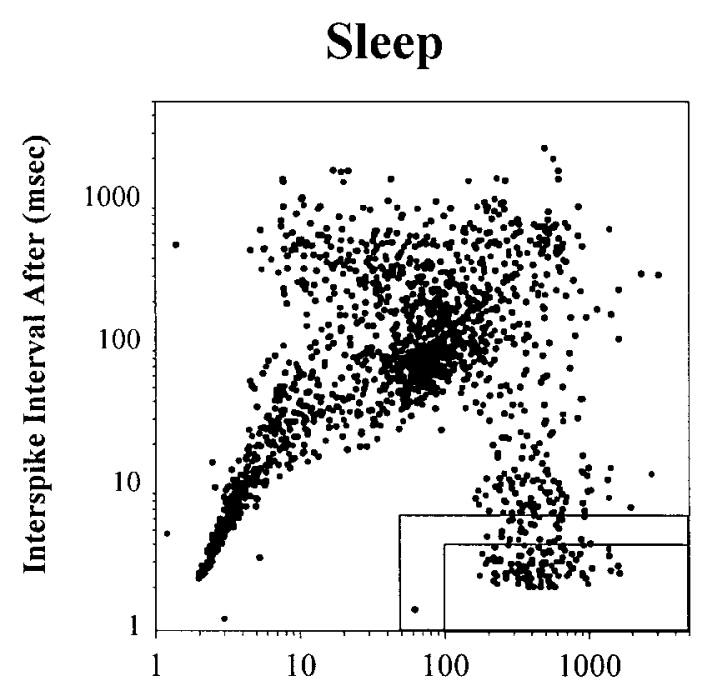

C

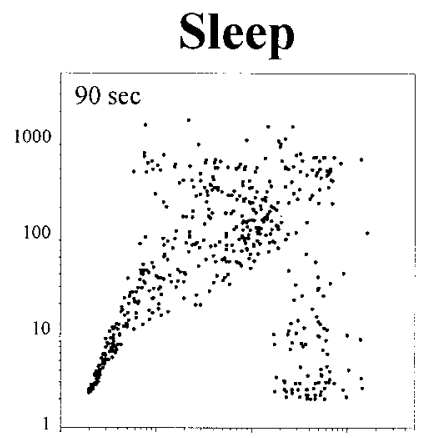

B
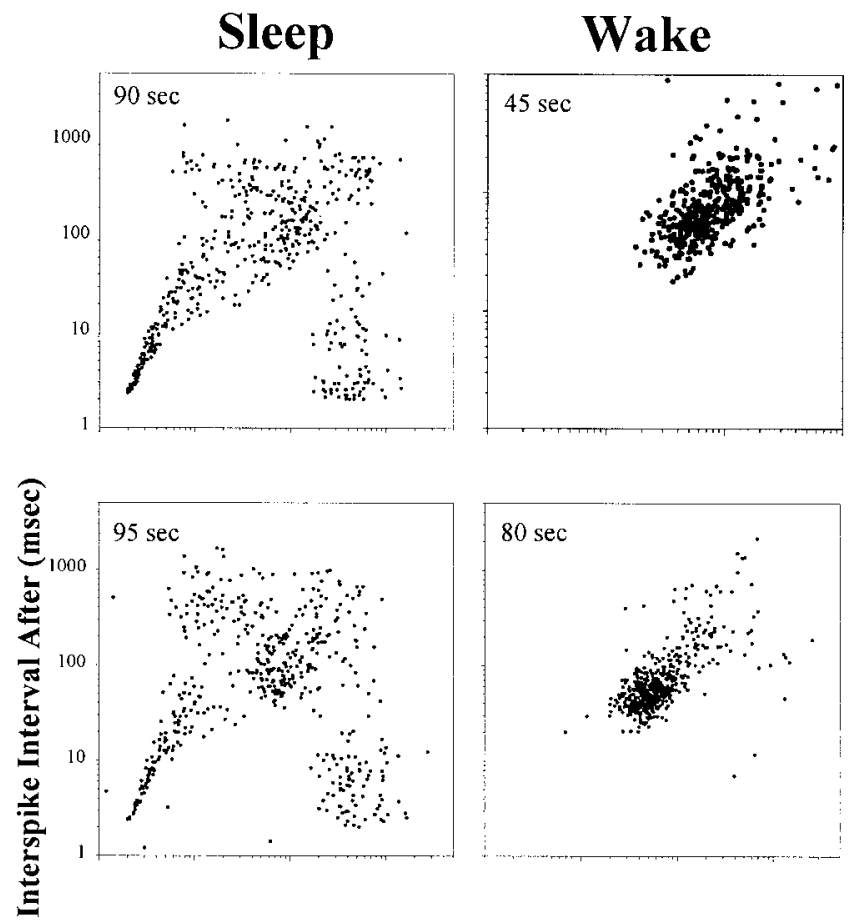

$80 \mathrm{sec}$
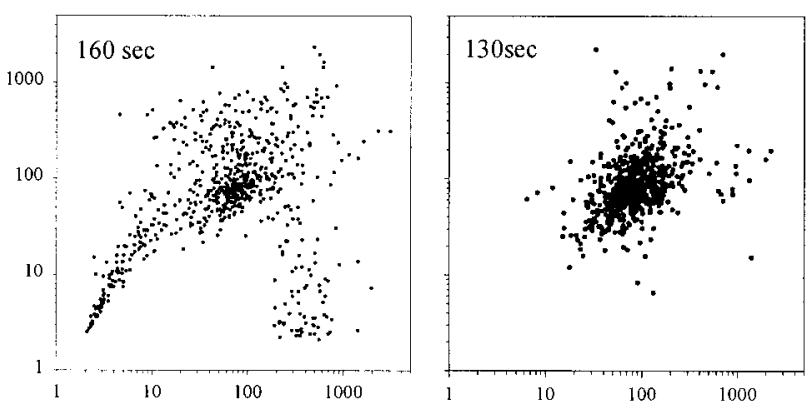

Interspike Interval Before (msec)

Interspike Interval Before (msec) 
low serve to emphasize that Figs. 1 and 2 are not necessarily representative. In fact, bursting during sleep is not ubiquitous, and tonic activity during wakefulness could be interrupted to include bursting.

Prevalence of bursting during SWS: how common is bursting during sleep?

Although bursting was common during sleep ( 0.51 bursts/s vs. 0.06 burst/s during waking, liberal criteria), the heterogeneity among LGN neurons in the degree to which they burst was striking. Figure $3 A$ presents the incidence of bursting among 57 LGN neurons during SWS. For this figure, bursting during each 5-s epoch of SWS is coded as a shade of gray, with black being zero bursts observed and white being five bursts or more. Figure $3 B$ presents the same data as in Fig. $3 A$, but now plotted as frequency of bursting during SWS. One-half of the cells failed to have a burst rate exceeding 0.5 bursts/s, and eight never burst. Another way of appreciating the heterogeneity in bursting among LGN neurons is that if we assume that these 57 neurons are representative, $50 \%$ of the bursting at any time is generated by $14 \%$ of the neurons.

As with burst frequency, the fraction of action potentials associated with bursting during sleep was appreciable, but variable. During some sleep epochs, $>70 \%$ of spikes were associated with bursting, whereas in several cells, bursting never occurred. Overall, we estimate $18 \%$ of spikes during slow-wave sleep were burst related. However, because we could not directly observe the $I_{\mathrm{T}}$ events, we are confident this is an underestimate (see DISCUSSION).

\section{Rhythmic bursting during SWS: how rhythmic is bursting during sleep?}

Rhythmic bursting is believed to play a prominent role in promoting the low-frequency EEG observed during SWS (e.g., McCormick and Bal 1997; Steriade et al. 1993). Although we showed that bursting is not ubiquitous among LGN neurons during SWS (Fig. 3), we did note that the presence of one burst increased the probability of observing another $(P<0.005$; based on comparing the observed median burst interval with 999 simulations in each of 17 LGN neurons). Figure $4 A$ shows the episodic nature of bursting (liberal criteria) in one LGN neuron over a 16 -min period during which the cat was mostly in SWS. Figure $4 B$ shows the distribution of interburst intervals for 17 LGN neurons for which we collected continuous records (10-50 $\mathrm{min})$ for various intervals of SWS. It indicates that $\sim 95 \%$ of the bursts occurred within $5 \mathrm{~s}$ of one another. To analyze potential underlying rhythmicity of such episodes, we

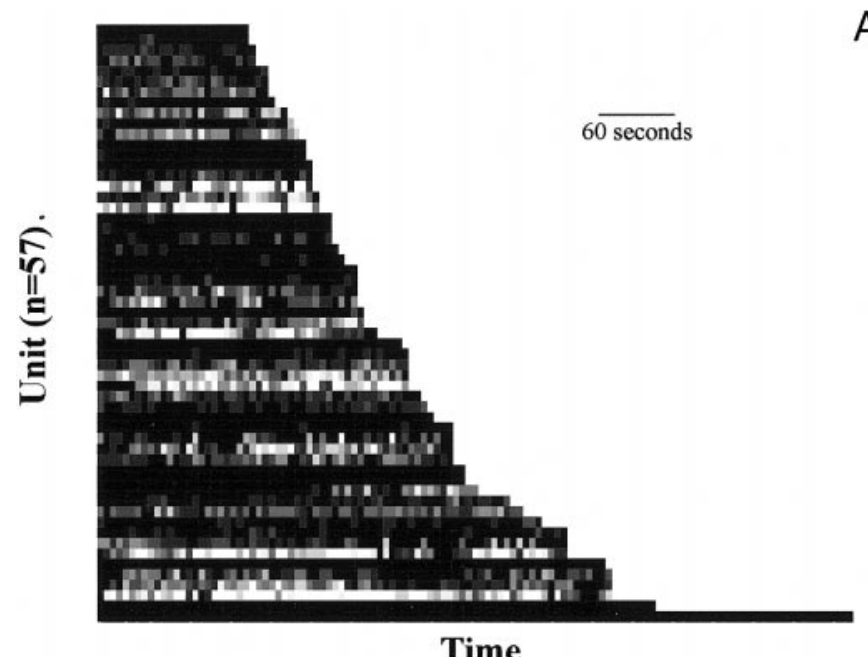

Time

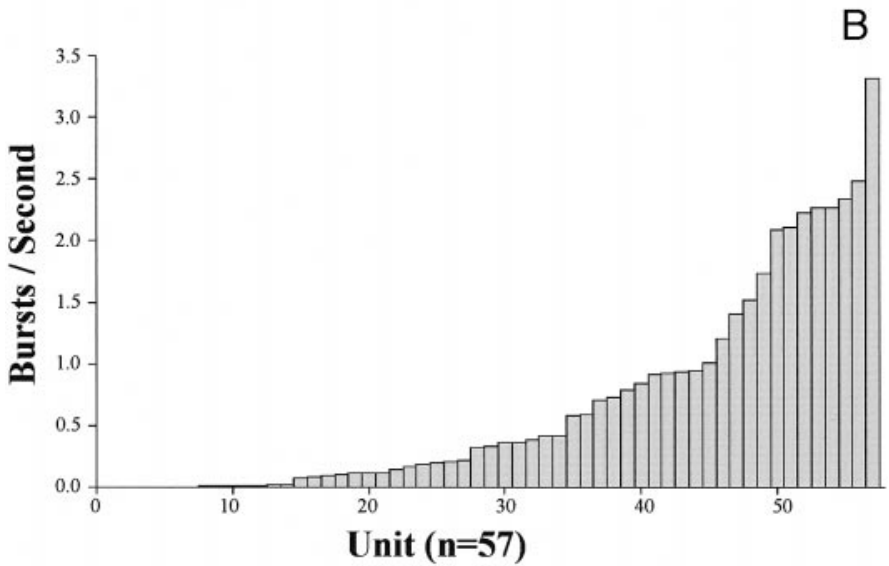

FIG. 3. Heterogeneity of bursting (liberal criteria) among thalamic neurons during slow-wave sleep. A: gray-scale representation of bursting activity during slow-wave sleep. Each horizontal strip shows the relative amount of bursting observed during sleep in an LGN cell. Each "patch" within the strip codes the number of bursts in a 5-s epoch, with black equal to zero and white equal to 5 or more bursts. The 57 neurons in this sample have been rankordered according to how much time was spent in slow-wave sleep. $B$ : ranking of the 57 neurons according to how frequently they burst.

identified "burst bouts" (see METHODS), i.e., periods during which bursting was common. If bursting was rhythmic, then the standard deviation of interburst intervals within a bout should approach zero. Figure $4 C$ shows two examples of autocorrellograms of two burst bouts, one highly rhythmic (Fig. 4C, top) and one arrhythmic (Fig. 4C, bottom). Figure $4 D$ shows the distribution of probabilities (determined using simulations; see METHODS) for 475 burst bouts collected from

FIG. 2. Two representations of LGN activity during sleep and waking. A: raster representation of activity during sleep and waking (marked by W and S labels on the right) over a 10-min period during which the cat drifted in and out of sleep. Each raster line represents $5 \mathrm{~s}$; there are $3 \mathrm{~s}$ between each line (not shown); small dots indicate action potentials, and large dots indicate bursts (liberal criteria). Note that bursting is confined to sleep. $B$ : the data presented in $A$ are presented in 2-dimensional joint-interval histograms (McCarley et al. 1983). The data have been divided into each of the 6 sleep or waking cycles observed. Each dot represents 1 action potential, and the location determined by the interval preceding (abscissa, log coordinates) and following (ordinate, log coordinates) the action potential. Duration of each epoch is indicated in the top left of each histogram. Note the regularity of activity during wakefulness (exhibited by tight clustering toward a single locus) vs. the descending "tails" during sleep. The clustering to the bottom right is the 1st spike in a burst, and the streaks to the bottom left are the subsequent spikes in a burst. $C$ : data collapsed from $B$ into just 2 joint-interval histograms (JIHs). The 2 insets in the bottom right of each JIH inscribe the cardinal spike in each burst using the liberal (larger inset, 6.66-ms interspike interval following $50 \mathrm{~ms}$ quiescence) or the conservative (smaller inset, 4-ms interspike interval following $50 \mathrm{~ms}$ quiescence) criteria used in this study. The liberal criteria increases the number of bursts during sleep by $\sim 25 \%$ and detects 1 burst (vs. 0 ) during wakefulness. 
A
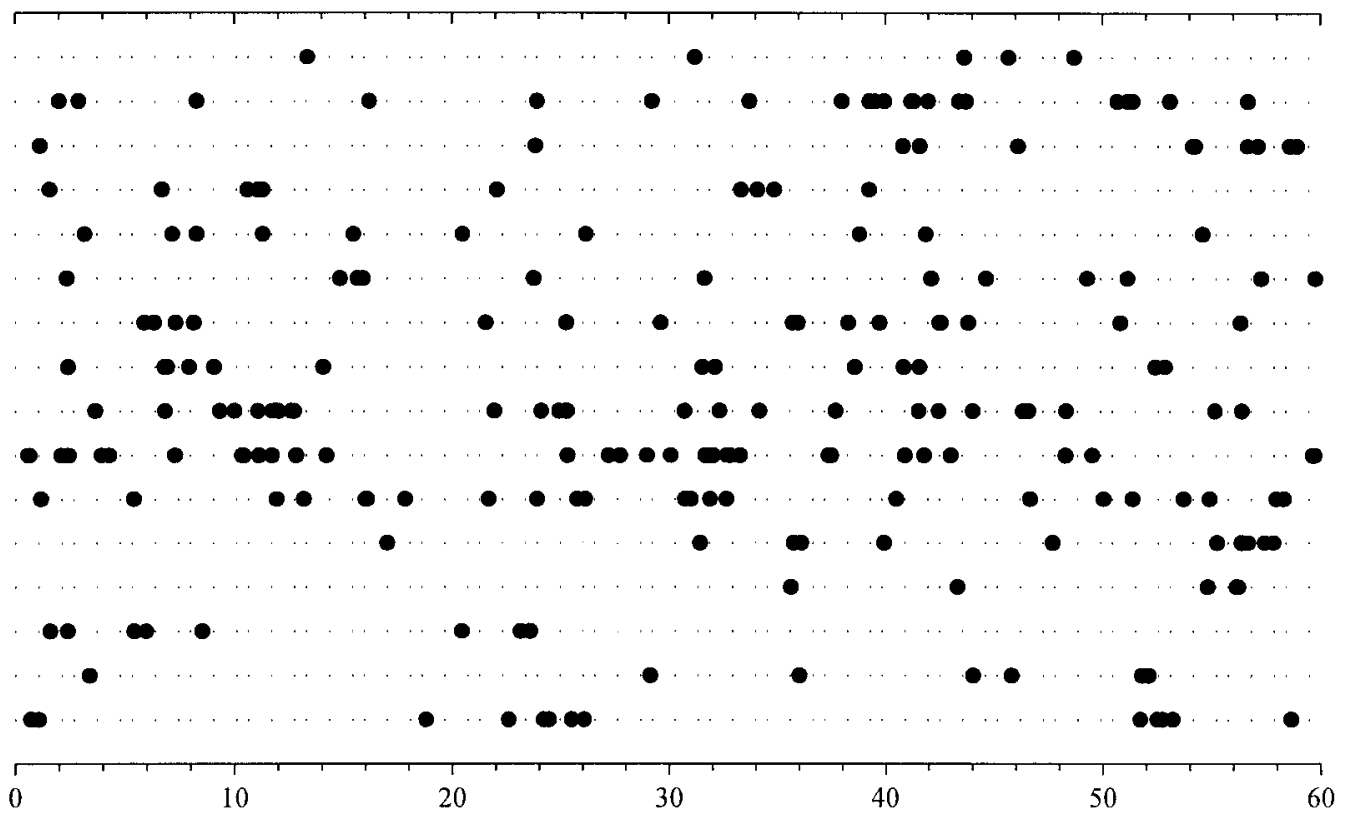

Time (sec)

B

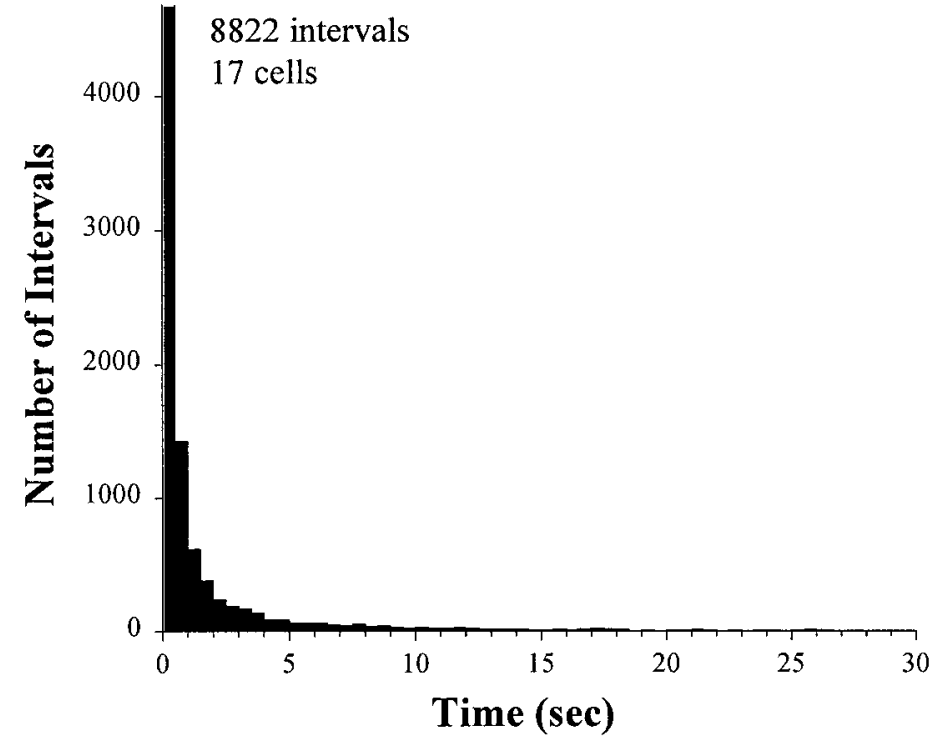

D

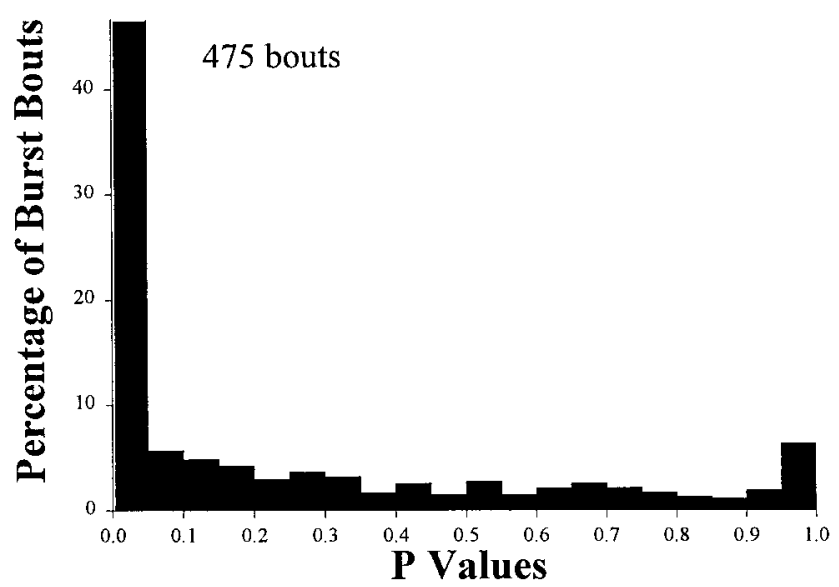

E
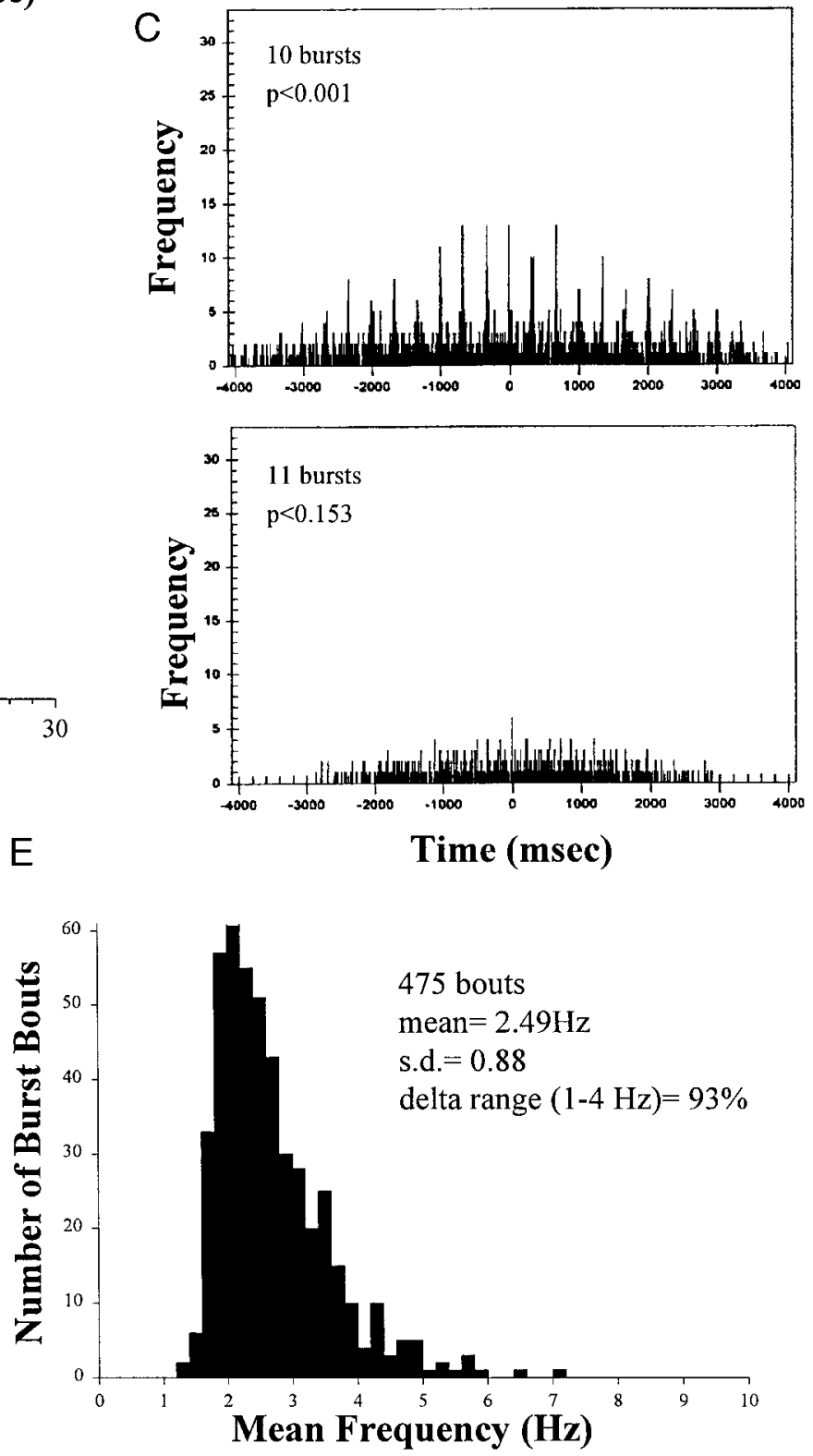
continuous records in the $17 \mathrm{LGN}$ neurons. Using a criterion of $P<0.05,47 \%$ of these bouts were rhythmic. Figure $4 E$ shows the distribution of interburst intervals, showing that the vast majority of intervals were concentrated within the delta (i.e., $0.5-4 \mathrm{~Hz}$ ) range. Although interburst intervals were within a frequency range appropriate for driving cortical delta rhythms, we failed to observe any consistent phase relationships between occipital EEG and concomitant thalamic bursting. This may, of course, be attributable to local variations in the occipital EEG (cf., McCarley et al. 1983).

\section{Bursting during visual stimulation: variability among neurons}

The majority of LGN neurons responded reliably and vigorously to visual stimuli of the appropriate polarity presented to the receptive field center. For most of these, bursting was not associated with the visually driven response. Figure $5 \mathrm{~A}$ shows the visually driven response of an LGN neuron in tonic mode to a flashing stimulus placed over the receptive field, whereas Fig. $5 B$ shows the visually driven response of a different LGN neuron whose initial response was often a high-frequency burst of action potentials (indicated by asterisks). Figure $5 C$ shows the burst probabilities for 93 LGN neurons evaluated under different conditions of visual stimulation. This figure emphasizes the heterogeneity among LGN neurons. For 33/93 neurons $(35 \%)$, we were unable to elicit any bursting, and as indicated, there was significant variability in bursting among the remaining 60 neurons. As with bursting during sleep, a minority of the neurons contributed most of the bursts. We found that $50 \%$ of the visually driven bursts originated from 9\% (8/93) of neurons. Finally, although it would not seem unreasonable that neurons that burst prolifically during visual stimulation would also burst extensively during sleep, such a relationship was not observed.

\section{Bursting during visual stimulation: stimulus size}

The probability of observing a burst as part of the visual response could be increased by increasing stimulus size. Figure $6 A$ shows the interspike interval histograms generated from single trials in which a $4^{\circ}$ stimulus (top) or a $40^{\circ}$ stimulus (bottom) are used to elicit the response. Clearly, bursting was evident with the large stimulus and not with the small stimulus. Figure $6 B$ shows JIHs for the responses when we used the $4^{\circ}$ stimulus (left) or the $40^{\circ}$ stimulus (right). Probability of bursting for the small stimulus was 0.01 bursts/cycle and 0.37 bursts/cycle for the large stimulus. Figure $6 C$ shows the probability of bursting we observed for each of four different stimulus sizes on this neuron. Table 1 shows that the results obtained for the single cell shown in Fig. 6 extend to a sample of 16 LGN neurons in which stimulus size was parametrically manipulated. For this larger sample, burst probability increased with stimulus size $\left(\chi^{2}=229, P<0.001,2\right.$ d.f. $)$.

\section{Bursting related to state}

The probability of bursting during visual stimulation was greatest for the initial stimulus cycle. Figure $7 A$ shows a single trial in which the initial, but not subsequent stimulus cycles elicited a burst. Figure $7 B$ shows the ordinal distribution of 319 bursts from 67 cells across the first 5 cycles of visual stimulation in each trial. Overall, if a visual stimulus was going to elicit a burst, the burst would most likely occur to the first stimulus presented in a trial $\left(\chi^{2}=17.01, P<0.005,4\right.$ d.f. $)$. One interpretation for this observation would be that the animal's attention increased as the visual stimuli were presented. The task demanded considerable attention in the sense that the cat must inhibit the prepotent reflex of looking at a newly presented stimulus in the field. In some trials, it is conceivable that, although the cat was "on target," the cat was less attentive and the cell relatively hyperpolarized.

Further evidence for a negative relationship between attention and bursting emerged when we analyzed bursting probability under "active" versus "passive" conditions. Figure 8 presents traces showing spike frequency during active (i.e., fixation spot present, Fig. 8A) and passive (i.e., no fixation spot present, Fig. $8 B$ ) conditions. As indicated by asterisks, bursts were present during six of the eight cycles shown under passive, but absent under active conditions. This cell was extensively analyzed under both active and passive conditions. During passive conditions, bursting occurred in 79/862 cycles $(9.2 \%)$, whereas only one burst (1/118 cycles, $0.8 \%)$ was observed under active conditions. This observation in the single cell could be extended to our overall sample, bursting was more common under active than passive conditions (Table 2, $\chi^{2}=109, P<0.001,1$ d.f., 65 neurons passive only, 14 neurons active only, 15 neurons active and passive).

Finally, the least-likely period during which a neuron would exhibit bursting appeared to be active fixation, with or without an overt fixation point. We analyzed 28 LGN neurons during which the cat maintained fixation on the dimmed laser point placed at the center of an otherwise blank screen. In some trials, this fixation point was extinguished for $1 \mathrm{~s}$ and then returned. Compared to an epoch of "spontaneous" activity taken immediately prior to trial onset, burst probability during fixation was nominally decreased $(0.046 / \mathrm{s}$ vs. $0.038 \mathrm{~s}$, n.s., conservative criteria). However, for each cell, the probability

FIG. 4. Bursting (liberal criteria) during sleep tended to be rhythmic. A: a 16-min continuous record of an LGN neuron indicating when bursting occurred (dots). For this record, the cat was mostly in slow-wave sleep, and bursting was only associated with sleep. Bursting tended to cluster in time. $B$ : compiled interval histogram showing 8,822 interburst intervals observed in 17 neurons that were continuously monitored. It shows that most interburst intervals were $1 \mathrm{~s}$ or less. $C$ : examples of autocorrellograms generated from a rhythmic burst-bout (top) and a poorly rhythmic burst-bout (bottom). The even spacing of the interburst intervals in the top autocorrellogram indicates rhythmicity. The results of the Monte Carlo simulations are plotted as probabilities for each burst-bout. For the top, the standard deviation of the observed interburst intervals was smaller than any of the simulations, and hence, $P<0.001$. For the bottom, 152 of the 999 simulations had standard deviations less than the observed standard deviation of interburst intervals, and hence, $P<0.153$. $D$ : histogram of the probabilities obtained from analyzing 475 burst bouts. Forty-seven percent (223) of the burst bouts showed a probability of 0.05 or less, indicating that nearly $1 / 2$ of all burst bouts during sleep are rhythmic, in a statistical sense. E: histogram plotting the mean frequency of each of the 475 burst bouts. The average frequency of these burst bouts was $2.49 \mathrm{~Hz}$ (i.e., average interburst interval was $\sim 400 \mathrm{~ms}$ ). As indicated, $93 \%$ of the interburst intervals were within the delta frequency range $(0.5-4 \mathrm{~Hz})$. 
A Visual stimulation: tonic firing

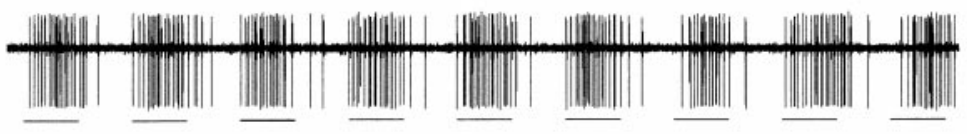

B Visual Stimulation: burst firing
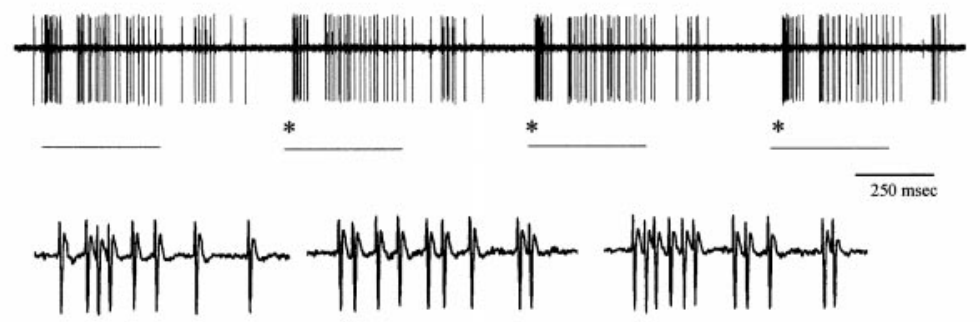

C

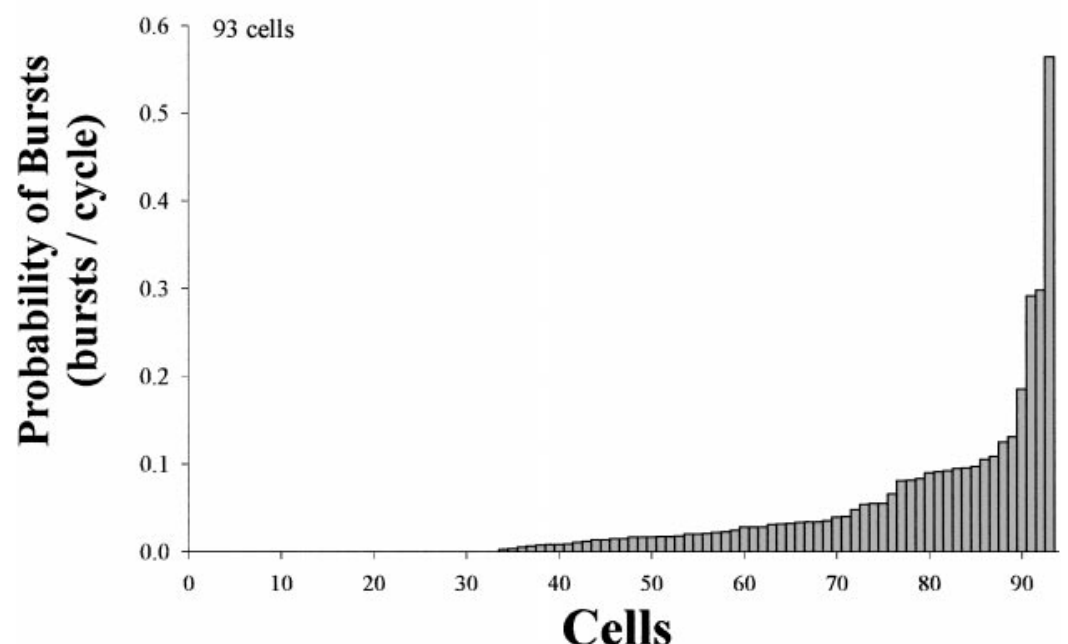

FIG. 5. Tonic and burst mode during visual stimulation. $A$ : tonic response of an LGN neuron when a $7^{\circ}$ square was flashed over the receptive field centered $11^{\circ}$ to the left of gaze, and on the horizon (bars indicate light part of stimulus cycle). B, top: different LGN neuron that showed burst responses (indicated by asterisks), when a rectangle $14^{\circ}$ wide by $12^{\circ}$ high was flashed over the receptive field centered $15^{\circ}$ left and $8^{\circ}$ up from gaze. Bottom: expanded traces of the 3 bursts. $C$ : distribution of burst probabilities for $93 \mathrm{LGN}$ neurons during visual stimulation. For each neuron, we divided the number of bursts associated with presentation of a visual stimulus by the total number of cycles. This figure represents the burst probabilities calculated from 11,212 visual responses. LGN neurons varied widely in the degree to which bursting represented part of the visual response. of observing a burst during the spontaneous epoch was greater than during fixation $\left(\chi^{2}=9.51, P<0.01,2\right.$ d.f.), reinforcing the view that membrane potential is depolarized during "attentive" states.

\section{I S C U S S I O N}

This study makes several contributions to our understanding of the burst/tonic dichotomy of LGN activity during sleeping and waking. First, the heterogeneity among LGN neurons in the degree to which they burst during either sleep or wakefulness had not been fully appreciated. Second, we were able to identify specific conditions within wakefulness in which burst probability increased dramatically. These conditions include presenting stimuli more likely to influence inhibitory surround mechanisms, as well as manipulating behavioral contingencies. The former observations reinforce the potency of the inhibitory surround in shaping visual response. The latter indicates that "wakefulness" can include shifts in membrane potential sufficient to de-inactivate the $I_{\mathrm{T}}$ burst mechanism. Finally, our method of analyzing potential rhythmic bursting indicates that most LGN neurons burst rhythmically during sleep, if only for a few seconds.
What is the structure of the thalamocortical network during sleep?

Several recent papers (e.g., see review by McCormick and Bal 1997) have promoted the idea that rhythmic bursting represents the dominant form of LGN activity during slowwave sleep. Rhythmic bursting was the exception, rather than the rule, in the previous study of activity in the cat's LGN (McCarley et al. 1983) (5 of 26 neurons). The recent study by Ramcharan et al. (2000) did not observe rhythmic bursting in the LGN of the sleeping monkey. We found that LGN bursting to be episodic, and rhythmic nearly half of the time. Although this seems to be a much different conclusion than the other studies, we strongly suspect that we were analyzing nearly identical spike trains. The difference is the method of analysis. McCarley et al. (1983) collapsed data across long epochs. Such treatment will necessarily dilute momentary rhythmicity that is interrupted by other arrhythmic epochs. The question McCarley et al. (1983) were addressing was whether there were overall trends in rhythmic behavior. The analysis Ramcharan et al. (2000) employed is straightforward: construct fast Fourier transforms (FFTs) from autocorrellograms and determine the statistical reliability of the FFT signal. Again, the problem is that they were analyzing long epochs that would dilute brief 
A

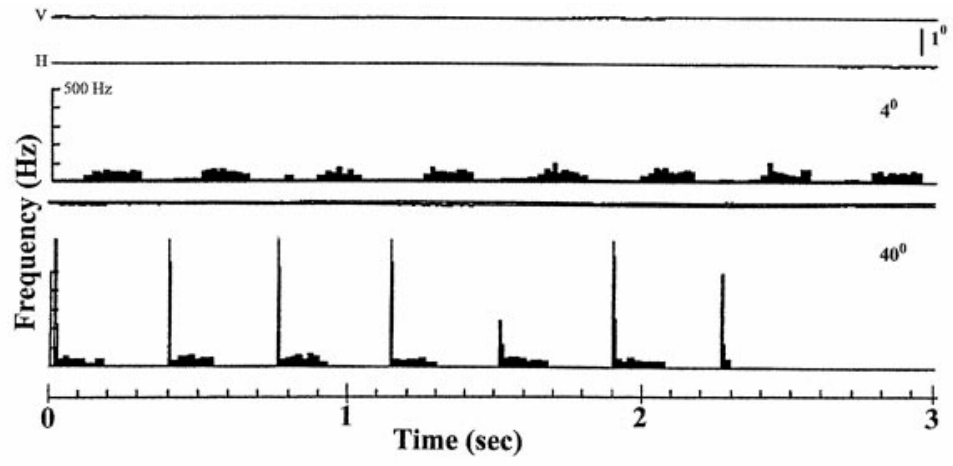

B

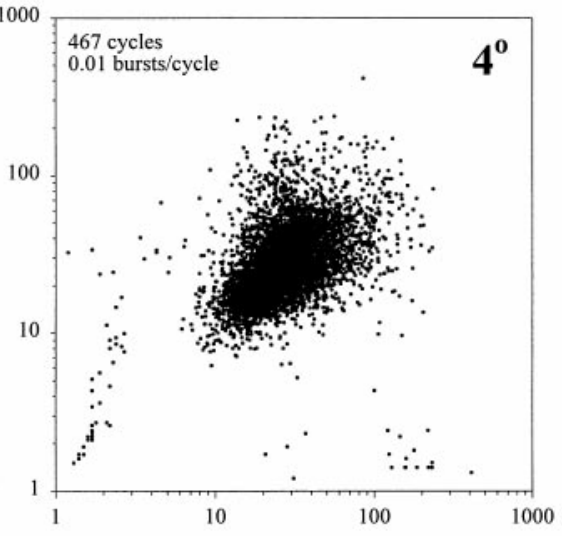

Interspike Interval Before (msec)

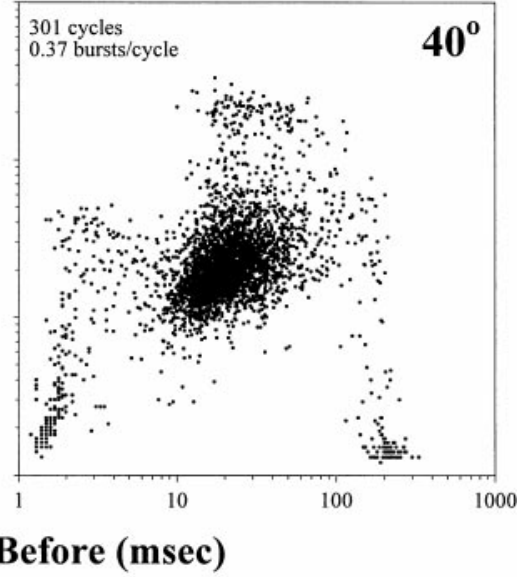

FIG. 6. Increased stimulus size increased burst probability. A: traces showing instantaneous spike frequency of an LGN neuron to presentation of a $4^{\circ}$ (top) and a $40^{\circ}$ flashing square (bottom). No bursting was observed with presentation of the small stimulus, whereas bursting was common with the large stimulus. $B$ : JIHs of the visual responses associated with the $4^{\circ}$ (left, 467 cycles) and $40^{\circ}$ (right, 301 cycles) stimulus. For this cell, burst probability was low with the small stimulus (0.01 bursts/ cycle) and relatively high (0.37 bursts/cycle) with large stimuli. $C$ : bar graph indicating burst probability increased with increased stimulus size $\left(\chi^{2}=229, P<0.001,2\right.$ d.f $)$. The numbers at the top of each bar indicate the total number of cycles evaluated.

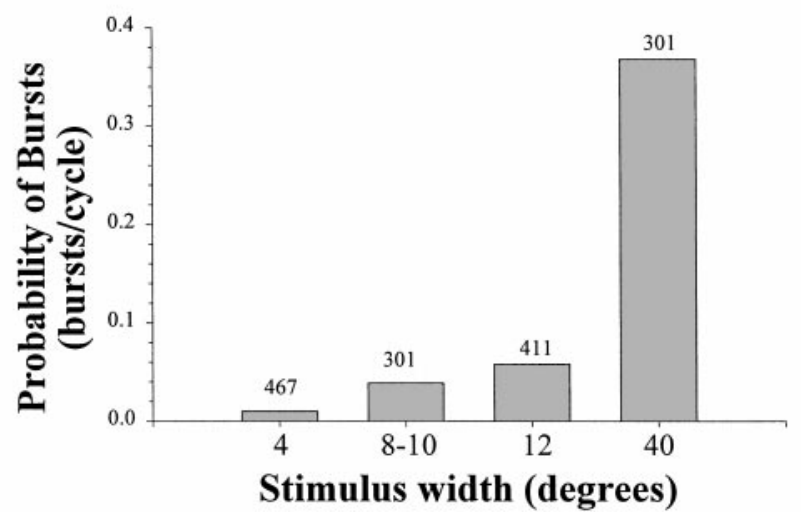

epochs of rhythmic behavior. Using an analysis that tests for rhythmicity over brief epochs, we found most LGN cells burst rhythmically, if only for a few seconds. Thus our observations support the idea that slow-wave sleep is a period of episodic disconnections from sensory inputs, allowing intrinsic conductances to manifest brief episodes of rhythmic activity (cf., McCormick and Bal 1997; Steriade et al. 1993). Finally, we know our measurements are conservative. Because we depend on extracellular spikes to imply an underlying $I_{\mathrm{T}}$ event, we have restricted our analysis to episodes of two spikes or more to identify bursting. In reality, there are $I_{\mathrm{T}}$ events that generate one or no spikes. Thus underlying rhythmic activity is almost certainly even more common.

We observed significant heterogeneity among LGN neurons in the degree to which they burst, whether during sleeping or waking. Although one way of interpreting our results is that during any given second in slow-wave sleep the probability of observing a burst in an LGN neuron is just under 28\%, such descriptions undermine the heterogeneity of the sample. Perhaps most telling is that $50 \%$ of the bursting appears to be produced by $14 \%$ of the neurons. McCarley et al. (1983) also noted heterogeneity in bursting among LGN neurons. They found a rather large fraction (37\%) that did not burst during slow-wave sleep (vs. 17\% for our large sample, 57 neurons). The functional significance of this heterogeneity is unknown. However, large populations of synchronous rhythmic bursting would not be desirable as such activity could easily incite epileptiform activity (cf., Steriade et al. 1993).

Variability in bursting among LGN neurons during sleep and wakefulness indicates that either LGN neurons vary in the degree to which the $I_{\mathrm{T}}$ channel is expressed, and/or, there are significant differences in intrinsic circuitry. Calcium imaging and whole cell in vitro studies suggest the $I_{\mathrm{T}}$ channel and its kinetics are homogenous among relay cells (Coulter et al. 
TABLE 1. Burst probability increases with stimulus size

\begin{tabular}{lrrrrrr}
\hline \hline & \multicolumn{5}{c}{ Stimulus Width } \\
\cline { 2 - 7 } & $0-5^{\circ}$ & $5-10^{\circ}$ & $10-15^{\circ}$ & $15-20^{\circ}$ & $20-25^{\circ}$ & $25-30^{\circ}$ \\
\hline Bursts & 71 & 100 & 49 & 148 & 130 & 50 \\
Cycles & 2,124 & 1,805 & 1,355 & 2,398 & 1,417 & 461 \\
Probability, bursts/cycle & 0.033 & 0.055 & 0.036 & 0.061 & 0.091 & 0.108 \\
\hline
\end{tabular}

1989; Hernandez-Cruz and Pape 1989; Munsch et al. 1997). Qualitative and quantitative differences exist among relay cell afferents. Besides obvious differences in $\mathrm{X}, \mathrm{Y}$, or $\mathrm{W}$ retinal afferents, there is evidence suggesting that intrinsic, brain stem, and corticothalamic afferents vary in their density and distribution (Friedlander et al. 1981; Murphy and Sillito 1996; Weber et al. 1989; Wilson et al. 1984). These differences in circuits offer an obvious avenue for heterogeneity. For example, while global variables such as sleep and waking might shift membrane polarity across large populations of neurons, local variations in cortical afferent activity could alter the probability of the specific neuron recorded expressing a burst.

Significant differences also exist between thalamic nuclei in the degree to which bursting is rhythmic during sleep. This point is explicit in the recent paper by Ramcharan et al. (2000). Despite an inability to observe rhythmic bursting in the monkey's LGN, rhythmic bursting was striking in the nearby ventrobasal complex. The observations by Ramcharan et al. (2000) in the ventrobasal complex appear consistent with the

A

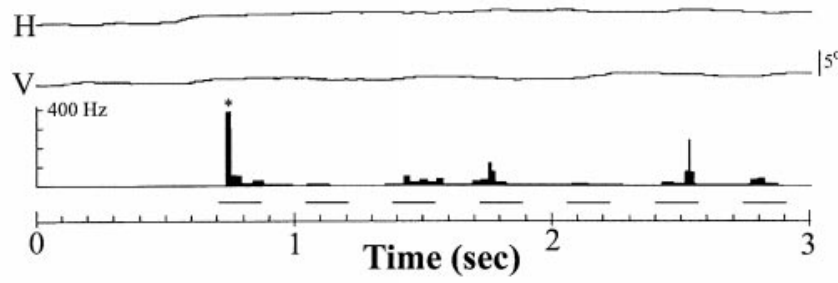

B

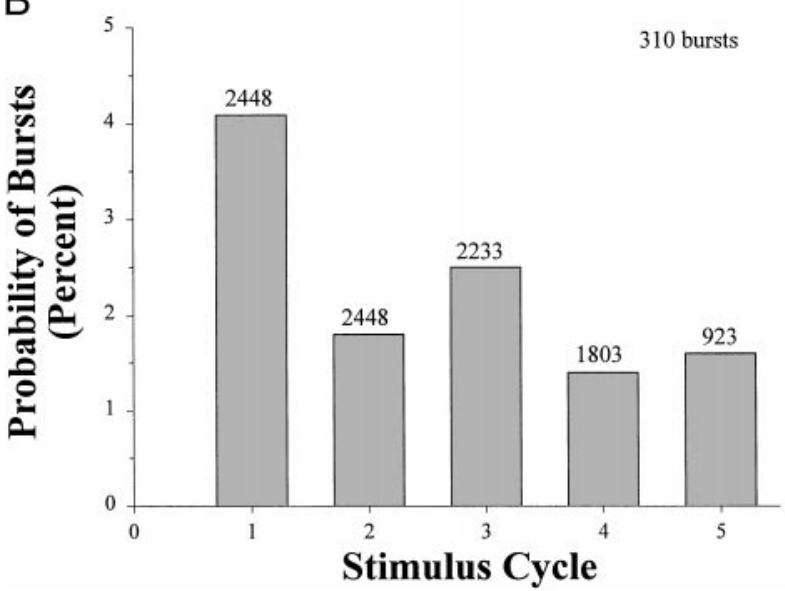

FIG. 7. Bursting was most prominent during the 1st cycle of visual presentation. A: shown are eye traces and instantaneous spike frequency records for a single trial in which presentation of a flashing rectangle $\left(5.4^{\circ} \times 5.0^{\circ}\right.$, ontime indicated by horizontal bars) over the receptive field initially elicits a burst (asterisk). B: bar chart indicating the frequency of bursting for each stimulus cycle $(9,855$ cycles for 60 LGN neurons) in which 2 or more cycles were presented. The number above each bar indicates how many cycles were analyzed. A burst was most likely observed on the 1st stimulus presentation. analyses offered by Steriade and his colleagues (Domich et al. 1986; Glenn and Steriade 1982) of highly rhythmic bursting in the cat's ventral lateral, ventral medial, and central lateral nuclei during slow-wave sleep.

\section{Sensory inputs, vigilance influence $I_{T}$ mechanism during wakefulness}

Activation of the $I_{\mathrm{T}}$ mechanism requires that the membrane potential be hyperpolarized for a period of 50-100 ms. This period allows the channel to "de-inactivate," such that subsequent depolarization will alter channel configuration allowing calcium entry. In agreement with previous studies, bursting is much less common during wakefulness than slow-wave sleep (Hubel 1960; Livingstone and Hubel 1981; McCarley et al. 1983; Ramcharan et al. 2000). The observation that bursting during wakefulness occurs at all may be somewhat surprising since the membrane potential is largely depolarized during this period (promoting the tonic mode). However, variations in state is only one contributor to altering conductances to alter membrane potential. The receptive fields of LGN neurons have an antagonistic center-surround organization in which stimuli of one polarity excite one portion of the field and inhibit the other. In the awake animal, this inhibition is capable of deinactivating the $I_{\mathrm{T}}$ channel, despite the prominence of a tonic mode. Coenen and Vendrik (1972) show analog traces in which an obvious burst is evident following the removal of inhibition by extinguishing a light presented to the center of an off-center LGN neuron. Bursting could be promoted during wakefulness by providing large stimuli that activated both center and surround mechanisms, by manipulating level of alertness, or, as previously documented, encouraging eye movements (Guido and Weyand 1995; Lee and Malpeli 1998). We interpret our results to simply indicate that during wakefulness membrane potential is dynamic, and each of the conditions that promoted bursting are conditions that would predictably hyperpolarize the membrane potential. Whereas membrane potential is generally more depolarized during wakefulness than during sleep (Hirsch et al. 1983), we were able to relatively easily produce bursts in many LGN neurons, indicating that the membrane could be hyperpolarized for extended periods. Each of the conditions under which we observed bursting are conditions when the membrane potential could be expected to be hyperpolarized.

Increasing the size of the visual stimulus increased burst probability. Given the center-surround organization of LGN neurons, increasing stimulus size should have a greater influence on the surround than smaller stimuli. For some LGN neurons, whole field illumination sufficiently hyperpolarizes the LGN neuron to de-inactivate the $I_{\mathrm{T}}$ channel and the occasional retinal input elicits a burst. In Fig. 6 we showed a more quantitative analysis of how stimulus size affects burst proba- 


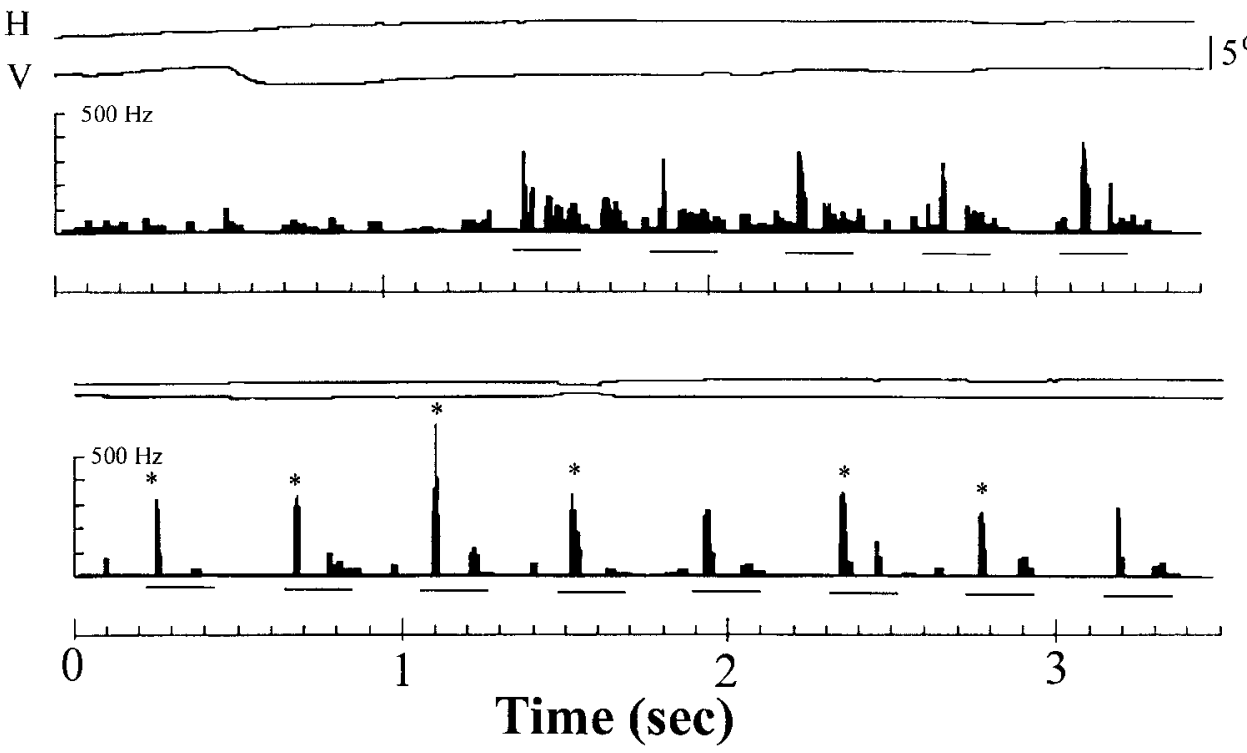

FIG. 8. Bursting was more common when visual stimulation was presented under "passive," rather than "active" conditions. Shown are eye traces and instantaneous spike frequency records for a trial in which the cat fixated on a spot as a rectangle $\left(12^{\circ} \times\right.$ $17^{\circ}$, bars indicate ontime) was presented over the receptive field (active, top), and a different trial in which gaze fortuitously aligned the visual stimulus over the receptive field (passive, bottom). Bursting (indicated by asterisks) represents the dominant response mode in the "passive" condition. Receptive field center for this neuron was $20^{\circ}$ left and $5^{\circ}$ up from gaze.

bility on a different LGN neuron. It is not obvious why large visual stimuli were more efficacious than small stimuli at eliciting bursts since our stimuli were sufficiently large in nearly all cases to include both the center and surround. One possibility is that the larger stimuli activated (inhibited) additional circuitry that lies beyond the classical receptive fields (e.g., McIlwain 1964), additionally hyperpolarizing the LGN neuron to deinactivate the $I_{\mathrm{T}}$ channel. Whatever the mechanism, the potency of large stimuli (over smaller stimuli) in elicting bursting has been observed previously in the LGN of the awake, paralyzed cat (Coenen and Vendrik 1972; their Fig. 2). Hyperpolarization associated with the large stimulus, but not the smaller stimulus, would be sufficient to de-inactivate the $I_{\mathrm{T}}$ channel that elicits bursting on the next cycle. Had we better control of gaze, it would have been interesting to determine how effective stimuli restricted to the surround such as annuli would have been in promoting bursting. Finally, although we could promote bursting by using large stimuli, it is worth emphasizing that large stimuli provided no guarantee of observing burst responses. One-third of neurons never burst during visual stimulation, and $50 \%$ of visually driven bursting originated from $9 \%$ of cells analyzed. This again serves to underscore the variability in response characteristics among LGN neurons.

State affects burst incidence because state affects membrane potential. This has been demonstrated directly by Hirsch et al. (1983), and indirectly by a number of investigators. Again, the records of Coenen and Vendrik (1972) are instructive. Following extinguishing a spot to the center of an oN LGN neuron, bursts could be observed in the "drowsy" state, but not in the "awake" state (Coenen and Vendrik 1972; Fig. 5). Such an observation indicates that the membrane potential can become

TABLE 2. Burst probability under passive and active gaze

\begin{tabular}{lrrr}
\hline \hline & Total & Passive & Active \\
\hline Bursts & 742 & 423 & 319 \\
Cycles & 17,744 & 6,869 & 10,875 \\
Probability, bursts/cycle & 0.042 & 0.062 & 0.029 \\
\hline
\end{tabular}

Number of neurons in Passive is 29 and in Active is 80 . sufficiently hyperpolarized to de-inactivate the $I_{\mathrm{T}}$ channel. Our own results, whether based on increased visually driven bursts associated with passive versus active gaze, or decreased burst probability associated with fixation, argues that state changes, as well as surround antagonism are capable of de-inactivating the $I_{\mathrm{T}}$ channel. Several investigators have commented on the increased activity and/or transfer ratio associated with alertness or arousal (Coenen and Vendrik 1972; Livingstone and Hubel 1981; Sakakura 1968; Swadlow and Weyand 1985; also reviewed by Sherman and Guillery 1996; Singer 1977).

\section{What is the significance of bursting during wakefulness?}

Having shown that bursting can occur during wakefulness and that such observations would be consistent with the known circuitry of the LGN, the question arises as to the functional significance of bursting in the awake animal. If bursting were restricted to slow-wave sleep, it and the supporting $I_{\mathrm{T}}$ channel could be viewed as an adaptation for establishing slow-wave sleep in cortex. In wakefulness, bursting is such a departure from a linear response; it is not clear what it means as a visual signal. One attractive idea is that bursting has little to do with analyzing visual detail, but instead serves as a "wake-up call" to cortex, switching the LGN from burst to tonic mode (Guido and Weyand 1995; Guido et al. 1995; Sherman 1996). As the animal's attention wanes, the neuron hyperpolarizes, de-inactivating the $I_{\mathrm{T}}$ channel. Subsequent visual stimuli would then activate the channel, sending a high-frequency volley of action potentials to visual cortex. This volley would be sufficiently powerful to drive corticothalamic neurons, which would then depolarize and switch LGN neurons to tonic mode via activation of metabotropic glutamate receptors (Godwin et al. 1996). In contrast to the burst mode, activity in the tonic mode is more linearly related to stimulus attributes (Guido et al. 1995; McCormick and Feeser 1990). Although attractive, our present results indicate that this idea is too limited. It does not explain, for example, the functional significance of bursting associated with saccades, nor with large visual stimuli. Further, as appealing and intuitive as the idea that bursting is more potent than single spike activity in activating cortical circuits (e.g., Lisman 
1997; Reinagel et al. 1999), empirical evidence in a thalamocortical system is lacking.

B. Renzi provided invaluable software support.

This research was supported by National Eye Institute Grant EY-R0111144.

\section{REFERENCES}

Bishop PO, BURKE W, AND DAVIS R. Single-unit recording from antidromically activated optic radiation neurones. J Physiol (Lond) 162: 432-450, 1962.

COENEN AML AND VENDRIK AJH. Determination of the transfer ratio of cat's geniculate neurons through quasi-intracellular recordings and the relation with the level of alertness. Exp Brain Res 14: 227-242, 1972.

Coulter DA, Huguenard JR, AND PRINCe DA. Calcium currents in rat thalamocortical relay neurons: kinetic properties of transient, low threshold current. J Physiol (Lond) 414: 587-604, 1989.

Domich L, OAKson G, and Steriade M. Thalamic burst patterns in the naturally sleeping cat: a comparison between cortically projecting and reticularis neurones. J Physiol (Lond) 379: 429-449, 1986.

FriedLander MJ, Lin C-S, Stanford LR, AND SHERMAN SM. Morphology of functionally identified neurons in lateral geniculate nucleus of the cat. J Neurophysiol 46: 80-129, 1981.

GlenN LL AND STERIADE M. Discharge rate and excitability of corticallyprojecting neurons in the intralaminar thalamic nuclei during waking and sleep states. J Neurosci 2: 1387-1404, 1982.

Godwin DW, VAughan JW, AND Sherman SM. Metabotropic glutamate receptors switch visual response mode of lateral geniculate nucleus cells from burst to tonic. J Neurophysiol 76: 1800-1816, 1996.

Guido W, Lu S-M, Vaughan JW, Godwin DW, and Sherman SM. Receiver operating characteristic (ROC) analysis of neurons in the cat's lateral geniculate nucleus during tonic and burst response mode. Vis Neurosci 12: 733-741, 1995

Guido W AND WeyAnd T. Burst responses in thalamic relay cells of the awake behaving cat. J Neurophysiol 74: 1782-1786, 1995.

HERNANDEZ-CRUZ A AND PAPE H-C. Identification of two calcium currents in acutely dissociated neurons from the rat lateral geniculate nucleus. $\mathrm{J} \mathrm{Neu}$ rophysiol 61: 1270-1283, 1989.

HiRsCh JC, Fourment A, AND MARC ME. Sleep-related variations of membrane potential in the lateral geniculate body relay neurons of the cat. Brain Res 259: 308-312, 1983.

HubeL DH. Single unit activity in lateral geniculate body and optic tract of unrestrained cats. J Physiol (Lond) 150: 91-104, 1960.

JudGe S, Richmond BJ, AND ChU FC. Implantation of magnetic search coils for measurement of eye position: an improved method. Vision Res 20: $535-538,1980$

LEE D AND MALPELI JG. Effects of saccades on the activity of neurons in the cat lateral geniculate nucleus. J Neurophysiol 79: 922-936, 1998.

LISMAN JE. Bursts as a unit of neural information: making unreliable synapses reliable. Trends Neurosci 20: 38-43, 1997.

LivingSTONE MS AND HuBEL DH. Effects of sleep and arousal on the processing of visual information in the cat. Nature 291: 554-561, 1981.

Lu S-M, GUIDO W, AND SHERMAN SM. Effects of membrane voltage on receptive field properties of lateral geniculate neurons in the cat: contributions of the low threshold $\mathrm{Ca}^{2+}$ conductance. J Neurophysiol 68: 2185-2198, 1992.

Lu S-M, Guido W, AND Sherman SM. The brain-stem parabrachial region controls mode of response to visual stimulation of neurons in the cat's lateral geniculate nucleus. Vis Neurosci 10: 631-642, 1993.
Malpeli JG, Weyand TG, and LaClair RL. A new method of mounting and directing chronically implanted microdrives. J Neurosci Methods 44: 19-26, 1992.

McCarley RW, Benoit O, AND BARRionuevo G. Lateral geniculate nucleus unitary discharge in sleep and waking: state- and rate-specific aspects. J Neurophysiol 50: 798-818, 1983.

McCoRMICK DA AND BAL T. Sleep and arousal: thalamocortical mechanisms. Aпnи Rev Neurosci 20: 185-215, 1997.

MCCORMICK DA AND FEESER HR. Functional implications of burst firing and single spike activity in lateral geniculate relay neurons. Neuroscience 39: 103-113, 1990.

MCILWAIN JT. Receptive fields of optic axons and lateral geniculate cells: peripheral extent and barbiturate sensitivity. J Neurophysiol 27: 1154-1173, 1964.

Munsch T, BudDE T, AND PAPE H-C. Voltage-activated intracellular calcium transients in thalamic relay cells and interneurons. Neuroreport 8: 24112418, 1997

MuRPHY PC AND SiLLito AM. Functional morphology of the feedback pathway from area 17 of the cat visual cortex to the lateral geniculate nucleus. J Neurosci 16: 1180-1192, 1996.

Ramcharan EJ, Gnadt JW, and Sherman SM. Burst and tonic firing in thalamic cells of unanesthetized, behaving monkeys. Vis Neurosci 17: $55-62,2000$

Reinagel P, Godwin D, Sherman SM, ANd Koch C. Encoding of visual information by LGN bursts. J Neurophysiol 81: 2558-2569, 1999.

RoBINSON DA. A method of measuring eye movement using a scleral search coil in a magnetic field. IEEE Trans Biomed Elect Eng 10: $137-145,1962$.

SAKAKURA H. Spontaneous and evoked unitary activities of cat lateral geniculate neurons in sleep and wakefulness. Jpn J Physiol 18: 23-42, 1968.

SHERMAN SM. Dual response modes in lateral geniculate neurons: mechanisms and functions. Vis Neurosci 13: 205-213, 1996.

SHERMAN SM AND GUILLERY RW. Functional organization of thalamocortical relays. J Neurophysiol 76: 1367-1395, 1996.

Sillito AM, Jones HE, Gerstein GL, AND West DC. Feature-linked synchronization of thalamic relay cell firing induced by feedback from the visual cortex. Nature 369: 479-482, 1994.

SINGER W. Control of thalamic transmission by corticofugal and ascending reticular pathways in the visual system. Physiol Rev 57: 386-420, 1977.

So YT and Shapley R. Spatial properties of X and Y cells in the lateral geniculate nucleus of the cat and conduction velocities of their inputs. Exp Brain Res 36: 533-550, 1979.

STERIADE M AND DESCHENES M. The thalamus as a neuronal oscillator. Brain Res Rev 8: 1-63, 1984.

SteRIADE M AND Llinas RR. The functional states of the thalamus and the associated neuronal interplay. Physiol Rev 68: 649-742, 1988.

Steriade M, McCormick DA, AND Sejnowski TJ. Thalamocortical oscillations in the sleeping and aroused brain. Science 262: 679-685, 1993.

SWAdLOW HA AND Weyand TG. Receptive field and axonal properties of neurons in the dorsal lateral geniculate nucleus of awake unparalyzed rabbits. J Neurophysiol 54: 168-183, 1985.

Weber AJ, Kalil RE, and Behan M. Synaptic connections between corticogeniculate axons and interneurons in the dorsal lateral geniculate nucleus of the cat. J Comp Neurol 289: 156-164, 1989.

WEYAND TG AND GAFKA AC. Activity of neurons in area 6 of the cat during fixation and eye movements. Vis Neurosci 15: 123-140, 1998.

WILSON JR, FRIEDLANDER MJ, AND SHERMAN SM. Fine structural morphology of identified X-and Y-cells in the cat's lateral geniculate nucleus. Proc $R$ Soc Lond B Biol Sci 221: 411-436, 1984 